\title{
WAVES AND PERSISTENCE IN MERGER AND ACQUISITION ACTIVITY
}

\author{
John T. Barkoulas \\ Department of Economics and Finance \\ Louisiana Tech University \\ Ruston, LA 71272 USA \\ Christopher F. Baum \\ Department of Economics \\ Boston College \\ Chestnut Hill, MA 02467 USA $^{1}$ \\ Atreya Chakraborty \\ Charles River Associates \\ Boston, MA 02116 USA
}

\begin{abstract}
Markov regime-switching and sine-wave models have been used to capture the apparent wave-like behavior in aggregate U.S. merger and acquisition (M\&A) activity. In this paper we offer an alternative characterization of the dynamic structure in M\&A activity as a strongly dependent or long-memory process.
\end{abstract}

Keywords: mergers, acquisitions, long memory, persistence JEL: G34, C12

1 Corresponding author. Tel. 1+617-552-3673, fax +1-617-552-2308, email baum@bc.edu. 


\section{Introduction}

Empirical evidence on the time series structure of aggregate merger and acquisition (M\&A) activity in the U.S. economy has attracted considerable attention in the literature. The question that frequently arises is whether merger activity occurs in "waves", that is, whether there are oscillations between low and high levels of merger activity. The answer to this question is important both in forecasting M\&A activity and in developing structural models of its stochastic behavior.

Recent research has made significant progress in defining "waves" and econometrically estimating the wave-like behavior in merger activity. The first formal testing of the merger wave hypothesis models aggregate merger activity as a two-state Markovswitching-regime model (Town, 1992). In this statistical framework, a merger wave is defined as a large, discrete increase in the mean of the series. Wave-like M\&A activity is consequently modeled as a combination of two AR processes with differing means with the transition from a high-wave (high-mean) state to a low-wave (low-mean) state based upon some probability law. Town finds that such a model fits M\&A time series behavior well, thus supporting the wave hypothesis.

Golbe and White (1993) provide a second direct test of merger wave behavior based on sine-curve estimations for the M\&A time series. They find that the coefficient estimate for the amplitude of the sine wave, which is the critical parameter for the wave hypothesis, is statistically significant, and thus conclude that the fitted model adequately describes the temporal patterns of aggregate merger activity. They hypothesize a cycle length for merger activity of about forty years based upon the value of the coefficient estimate for the period of the wave.

Contrary to Golbe and White's model, merger activity exhibits stretches of high and low values with no obvious hidden or strict periodicity. These temporal patterns of cyclic but nonperiodic behavior appear to be distinct phenomena that embody essential aspects of the data. We argue that this tendency to observe groups of high or low values in merger 
activity is consistent with an underlying data generating process exhibiting long memory or strong dependence. ${ }^{2}$ A long-memory process can give rise to such stretches, or waves, which occur without any regularity in either time of occurrence or duration. If a time series exhibits the long-memory (long-term dependence) property, persistent temporal dependence exists even between distant observations. Such series exhibit hyperbolically declining autocorrelations and low-frequency peaks in their spectral distribution. If long memory is a feature of the temporal behavior of M\&A activity, a shock to M\&A activity persists for a long time, even though it eventually dissipates.

To uncover persistent dependence in M\&A activity, we estimate the long-memory or fractional-differencing parameter using the Gaussian semiparametric and exact maximum likelihood methods. The time series investigated tracks M\&A activity in the U.S. over a long time period, covering approximately a century of data. The length of the sample period, and not merely the sample size, is important when modeling the long-run dynamics of a time series. We establish strong and robust evidence of long-term dependence in the M\&A time series, therefore supporting our alternative characterization of the dynamic structure in M\&A activity. This evidence of long memory for the merger series remains even after accounting for potential nonstationarities in the mean level of the series, further reinforcing that strong dependence is a genuine feature of the random function underlying the stochastic behavior of merger activity. We offer possible explanations for the presence of long-memory dynamics in merger activity at both theoretical and empirical levels.

The rest of the paper is constructed as follows. Section 2 presents the fractionaldifferencing methods employed. Data, empirical results, and discussion of their implications are presented in Section 3. We summarize and conclude in Section 4.

\footnotetext{
2 This behavior has been called the "Joseph effect" by Mandelbrot and Wallis (1968) in reference to the Old Testament prophet who foretold of the seven years of plenty followed by the seven years of famine that Egypt was to experience.
} 


\section{Fractional Differencing Estimation Methods}

The model of an autoregressive fractionally integrated moving average process of order $(p, d, q)$, denoted by ARFIMA $(p, d, q)$, with mean $\mu$, may be written using operator notation as

$$
\Phi(L)(1-L)^{d}\left(y_{t}-\mu\right)=\Theta(L) u_{t}, u_{t} \sim \text { i.i.d. }\left(0, \sigma_{u}^{2}\right)
$$

where $L$ is the backward-shift operator, $\Phi(L)=1-\phi_{1} L-. .-\phi_{p} L^{p}, \Theta(L)=1+\vartheta_{1} L$ $+\ldots+\vartheta_{q} L^{q}$, and $(1-L)^{d}$ is the fractional differencing operator. The parameter $d$ is allowed to assume any real value. The arbitrary restriction of $d$ to integer values gives rise to the standard autoregressive integrated moving average (ARIMA) model. The stochastic process $y_{t}$ is both stationary and invertible if all roots of $\Phi(L)$ and $\Theta(L)$ lie outside the unit circle and $|d|<0.5$. The process is said to exhibit long-memory behavior if $d \in(0,1)$. For $d \in[0.5,1), y_{t}$ is nonstationary (having an infinite variance) but it is mean-reverting.

We estimate the long-memory parameter using Robinson's Gaussian semiparametric approach and Sowell's exact maximum likelihood method. Robinson (1995) proposes a Gaussian semiparametric estimator, GS hereafter, of the self-similarity parameter $H$. Assume that the spectral density of the time series, denoted by $f(\cdot)$, behaves as

$$
f(\xi) \sim G \xi^{1-2 H} \text { as } \xi \rightarrow 0^{+}
$$

for $\mathrm{G} \in(0, \infty)$ and $H \in(0,1)$. The self-similarity parameter $H$ relates to the longmemory parameter $d$ by $H=d+\frac{1}{2}$. The estimate for $H$, denoted $\widehat{H}$, is obtained through minimization of the function

$$
R(H)=\ln \widehat{G}(H)-(2 H-1) \frac{1}{\nu} \sum_{\lambda=1}^{\nu} \ln \xi_{\lambda}
$$

with respect to $H$, where $\widehat{G}(H)=\frac{1}{\nu} \sum_{\lambda=1}^{\nu} \xi_{\lambda}^{2 H-1} I\left(\xi_{\lambda}\right)$. The GS estimator is $\nu^{\frac{1}{2}}$-consistent 
with a variance of the limiting distribution free of nuisance parameters and equal to $\frac{1}{4 \nu}$.

Sowell's (1992a) exact maximum likelihood (ML) method allows for the simultaneous estimation of both the long-memory and ARMA parameters. Assuming normality of the innovations in (1), the log likelihood function for the sample of $T$ observations is given by

$$
L\left(\gamma ; Y_{T}\right)=-\frac{T}{2} \log (2 \pi)-\frac{1}{2} \log \left|\Sigma_{T}\right|-\frac{1}{2}\left(Y_{T}^{\prime} \Sigma_{T}^{-1} Y_{T}\right)
$$

The ML estimator, which is obtained by maximizing (4) with respect to the parameter vector $\gamma=\{\Phi, \Theta, d\}$, is consistent and asymptotically normal.

\section{$3 \quad$ Data and Empirical Findings}

The data series, constructed by Town (1992), consists of quarterly observations on M\&A activity in the U.S., as measured by the frequency of deals, covering the period 1895:1 to 1989:1 for a total of 377 observations. ${ }^{3}$ The graph of aggregate M\&A activity, shown in Figure 1, clearly displays a wave-like behavior with several notable peaks. The most intense quarter of M\&A activity is 1899:1 while the least intense quarter occurred during the Great Depression (1932:1). Over time, it appears that both the duration and amplitude of merger waves have declined. There are significant departures from normality as the series is positively skewed and leptokurtic. ${ }^{4}$ There is no evidence of conditional volatility in the series based on Engle's Lagrange multiplier test. ${ }^{5}$

The primary motivation for testing for fractional roots in the merger series is the nature of cyclic behavior in the data. The observed behavior of stretches of high and low merger activity without any regularity in the time of occurrence and/or duration suggests an underlying strongly dependent data generating process. Fractional exponent

\footnotetext{
3 We thank R. Town for providing us with his data sets. See Town (1992) for more details on the construction of the data series.

4 The skewness and excess kurtosis coefficients are 2.264 (0.000) and 7.180 (0.000), respectively.

5 The test statistic for ARCH effects of order four is $4.479(0.345)$.
} 
estimates based on the GS method for the level series are reported in Table 1. The $d$ estimates obtained are significantly positive and stable over estimation sample sizes, thus suggesting robustness. Table 2 reports ARFIMA estimation results based on Sowell's exact ML estimation method. We report $d$ estimates for various ARFIMA $(p, d, q)$ models, with the maximum order allowed in the AR and MA polynomials being three: $p \leq 3$ and $q \leq 3$. The exact ML $d$ estimates are significant and stable across the various orders of the short-memory (ARMA) structure. Even though the SIC and AIC criteria select different ARFIMA specifications, their long-memory parameters are very close in magnitude. The exact ML $d$ estimates are similar in value to the GS estimates, with a value around 0.6. Merger activity is characterized by a great deal of persistence.

To account for the potential bias-inducing effects of neglecting one or more mean shifts in the M\&A series, we prefilter the series by regressing it on a constant and a set of dummy variables corresponding to periods of intense merger activity. ${ }^{6}$ We assume these periods to be exogenously given, as identified in Town (1992, p.S97). He identifies nine such periods, with the following dating: 1898:1-1902:4, 1919:2-1921:4, 1925:3-1932:2, 1945:4-1946:1, 1954:3-1955:3, 1960:1-1960:2, 1962:1-1962:2, 1967:2-1969:4, and 1986:4. A similar procedure to deal with potential in-mean nonstationarities when testing for long memory is followed in Cheung (1993).

The GS estimates of the long-memory parameter for the prefiltered M\&A time series are reported in Table 1. These estimates are positive, stable across estimation sample sizes, and statistically significant. The long-memory estimates for the prefiltered M\&A time series are lower in value than the original ones, suggesting the possibility of an upward bias initially due to potential switching dynamics in the mean of the process. Similar evidence is obtained based on the exact ML method. As the last column of Table 2 reports, both SIC and AIC criteria select ARFIMA models with significantly positive fractional-differencing parameters which are very similar in magnitude. Both estimation methods produce estimates of the long-memory parameter around the value of 0.20 . Although the estimated value of the fractional parameter is significantly reduced,

6 Cheung (1993) shows that long-memory tests are robust to nonstationarities in the variance, but biased toward finding long memory $(d>0)$ in the presence of frequent shifts in the mean of the process. 
evidence of long memory in merger activity remains even after accounting for potential inmean nonstationarities. The obtained long-memory evidence is therefore non-spurious, in that it is an endogenous phenomenon of the M\&A series dynamics. Shocks to the merger series have a long-lasting effect; the correlations of the series are not summable. For all practical purposes, merger activity may be considered to have an infinite span of statistical interdependence.

Our findings of persistence are consistent with previous theoretical and empirical research on the structural relationship between aggregate merger activity and macroeconomic factors. Theoretically, our findings are consistent with Gort's (1969) view that "economic disturbances" cause sudden change in the frequency in M\&A activity. These "disturbances," Gort argues, increase the variance of forecasts of a firm's future profitability, causing divergence in firm valuation between shareholders and non-shareholders. If the means of both the overall distribution of firm values and that of current shareholders change by the same proportion, the likelihood that current shareholders may undervalue their firm relative to a group of potential acquirers is increased, thus triggering a wave of merger activity. Key to this logic is the assumption that dispersion of the forecasts (regarding firm profitability) made from post-disturbance information is greater than those that use pre-disturbance information. This framework suggests that substantial amount of post-disturbance information would have to accumulate before past information will be a reliable predictor for the future. Hence, there may be long periods of time where sizable discrepancies in valuation between stockholders and non-stockholders may remain, fueling the observed persistence of $M \& A$ activity.

On the empirical level, long-memory dynamics in merger activity may reflect the statistical properties of fundamental factors underlying the series: merger activity may be generated by an economic process that contains long-term dependence. Some of the proposed determinants of M\&A activity which have been shown to be statistically significant in previous research are stock prices, wholesale commodity prices, nominal interest rates, real interest rates, and risk and term premia in bond yields. Several of these determinants of M\&A activity have been shown to exhibit persistence. More 
specifically, fractal structure has been found in GNP (Sowell (1992b)), bond yields (Shea (1991)), interest-rate differentials (Baillie and Bollerslev (1994)), and real interest rates (Diebold and Lindner (1996)).

\section{Conclusions}

In this paper we provide an alternative characterization of U.S. M\&A activity as a strongly autocorrelated process. The observed nonperiodic cycle in the time series of merger activity can be attributed to the presence of long-memory dynamics. This evidence of long-term dependence is robust to the estimation method employed and the potential presence of shifts in the mean of the series, thus suggesting that long memory is a genuine, essential feature of the data. Modeling merger activity as a long-range dependent process provides insights into the persistence of shocks, which dissipate at the slow hyperbolic rate of decay. Movements in merger activity appear to be influenced not only by their recent history but also by realizations from the distant past. Persistence in merger activity is consistent with Gort's (1969) "economic disturbance" theory and, at the empirical level, may reflect the statistical properties of the fundamental factors driving the series' dynamic behavior. 


\section{References}

[1] Baillie, R. and T. Bollerslev (1994), Long memory in the forward premium, Journal of International Money and Finance, 13, 565-571.

[2] Cheung, Y. W. (1993), Tests for fractional integration: A Monte Carlo investigation, Journal of Time Series Analysis, 14, 331-345.

[3] Diebold, F. X. and P. Lindner (1996), Fractional integration and interval prediction, Economics Letters, 50, 305-313.

[4] Golbe, D. and L. White (1993), Catch a wave: The time series behavior of mergers, Review of Economics and Statistics, 493-499.

[5] Gort, M. (1969), An economic disturbance theory of mergers, Quarterly Journal of Economics, 83, 624-642.

[6] Mandelbrot, B. and J. R. Wallis (1968), Noah, Joseph and operational hydrology, Water Resources Research, 909-918.

[7] Robinson, P. (1995), Gaussian semiparametric estimation of long range dependence, Annals of Statistics, 13, 1630-1661.

[8] Shea, G. S. (1991), Uncertainty and implied variance bounds in long-memory models of the interest rate term structure, Empirical Economics, 16, 287-312.

[9] Sowell, F. (1992a), Maximum likelihood estimation of stationary univariate fractionallyintegrated time-series models, Journal of Econometrics, 53, 165-188.

[10] Sowell, F. (1992b), Modeling long-run behavior with the fractional ARIMA model, Journal of Monetary Economics, 29, 277-302.

[11] Town, R. J. (1992), Merger waves and the structure of merger and acquisition timeseries, Journal of Applied Econometrics, 7, S83-S100. 


\section{Table 1: Gaussian Semiparametric Estimates of the Fractional Parameter $d$ for the Level and Prefiltered M\&A Time Series, 1985:1-1989:1

$\begin{array}{cccc}\text { Dependent Variable } & d(0.55) & d(0.575) & d(0.60) \\ \text { Level Series } & 0.581(5.925)^{* * *} & 0.614(6.726)^{* * *} & 0.610(7.217)^{* * *} \\ \text { Prefiltered Series } & 0.215(2.192)^{* *} & 0.269(2.946)^{* * *} & 0.279(3.301)^{* * *}\end{array}$

Notes: The M\&A time series is that constructed by Town (1992). The prefiltered M\&A time series is obtained by regressing the original series on a constant and a set of dummy variables corresponding to time periods with intense merger activity as identified by Town (1992). $d(0.55), d(0.575)$, and $d(0.60)$ give the $d$ estimates corresponding to sample sizes $\nu=T^{0.55}, \nu=T^{0.575}$, and $\nu=T^{0.60}$. The superscripts ***, **, * indicate statistical significance for the null hypothesis $d=0$ against the alternative $d \neq 0$ at the $1 \%, 5 \%$, and $10 \%$ levels, respectively. 
Table 2: Maximum Likelihood Estimates of the Fractional Parameter $d$ for the Level and Prefiltered M\&A Time Series, 1985:1-1989:1

$\begin{array}{cc}\text { ARMA }(p, q) & \\ \text { Order } & \text { LEVEL SERIES } \\ & \\ & \\ (0,0) & 0.614^{a}(14.423) * * * \\ (0,1) & 0.650(8.298) * * * \\ (0,2) & 0.568(6.102) * * * \\ (0,3) & 0.631(5.352) * * * \\ (1,0) & 0.656(9.558) * * * \\ (1,1) & 0.653^{b}(15.304) * * * \\ (1,2) & 0.578(7.273) * * * \\ (1,3) & 0.645(5.488) * * * \\ (2,0) & 0.553(6.936) * * * \\ (2,1) & 0.586(14.435) * * * \\ (2,2) & 0.591(8.188) * * * \\ (2,3) & 0.648(5.538) * * * \\ (3,0) & 0.835(6.865) * * * \\ (3,1) & 0.728(5.717) * * * \\ (3,2) & 0.756(8.893) * * * \\ (3,3) & 0.682(7.886) * * *\end{array}$

Long Memory parameter $d$

$\left(t-\right.$ statistic for $\left.H_{0}: d=0\right)$

Prefiltered SERIES

$0.416(11.681) * * *$

$0.297(4.724) * * *$

$0.173(2.834) * * *$

$0.237(2.257) * * *$

$0.200^{a}(2.023) * * *$

$0.169(1.385)$

$0.234(3.372) * * *$

$0.198(3.916) * * *$

$0.164(1.272)$

$0.172^{b}(3.659) * * *$

$0.192(1.780) * * *$

$0.219(3.137) * * *$

$0.267(2.166) * * *$

$0.201(1.904) *$

$0.207(9.320) * * *$

$0.218(6.375) * * *$

Notes: See notes in Table 1 for data details. The ML method is the exact maximum likelihood method proposed by Sowell (1992a). ***, **, * indicate statistical significance for the null hypothesis $d=0$ against the alternative $d \neq 0$ at the $1 \%, 5 \%$, and $10 \%$ levels, respectively. a (b) indicates that the particular ARFIMA model was chosen according to the SIC (AIC) model selection criterion. 
Figure 1: Merger \& Acquisition Time Series, 1895:1-1989:1

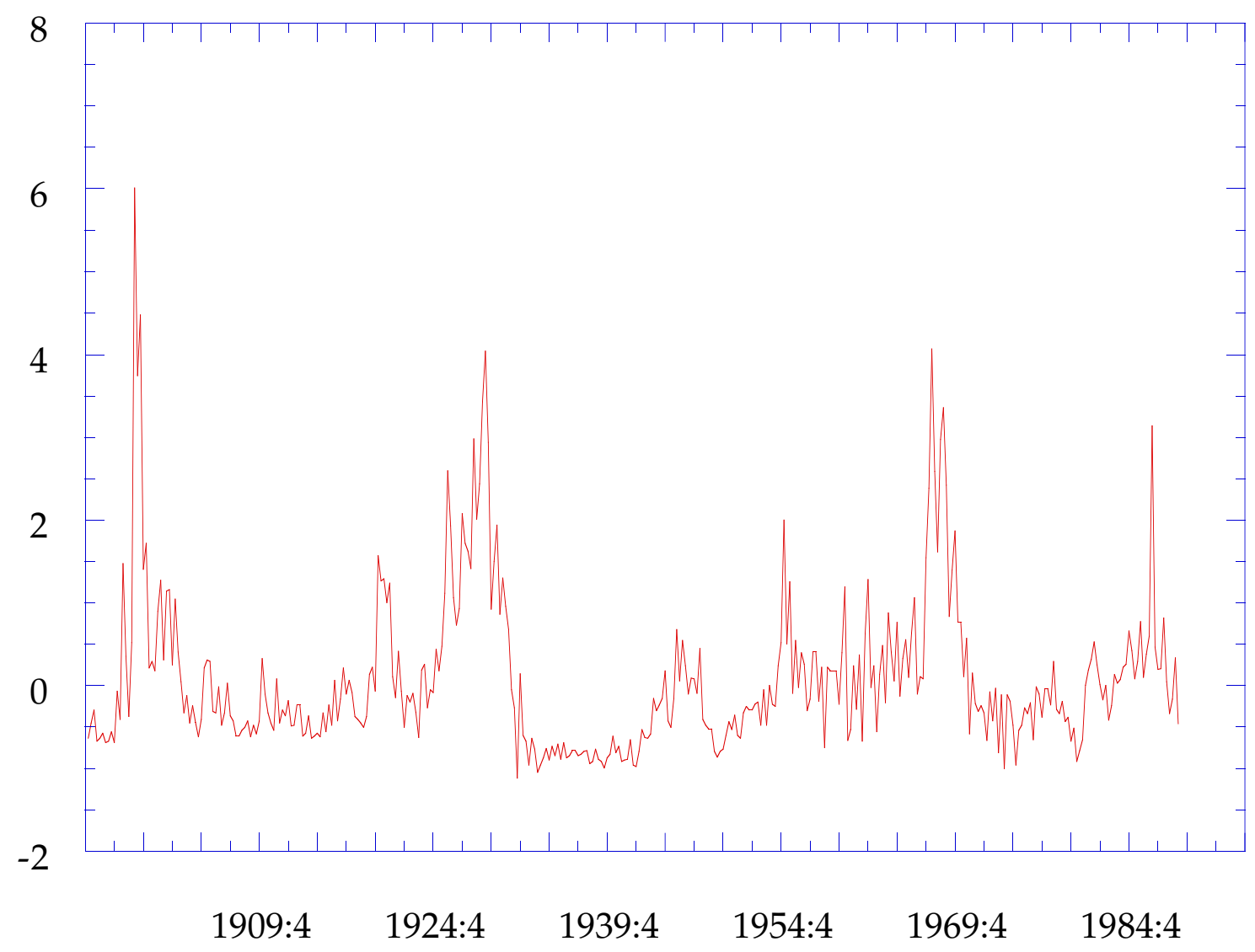

